\title{
Pearls
}

\section{Pearls: Snakes in the Grass and Other Surgical Gems}

\author{
Amy L. Ladd MD
}

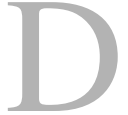
isease, injury, and other conditions deprive the human body of its well-timed machinery and intricate beauty. It is our privilege as surgeons to assist in its salvage and rehabilitation. Surgical procedures have a beginning, a middle, and an end. Preparation, surgical execution, and wound closure with

Note from the Editor-in-Chief:

We are pleased to present to readers of Clinical Orthopaedics and Related

Research ${ }^{\circledR}$ the next installment of "Pearls". In this column, distinguished surgeons, scientists, or scholars share surgical or professional tips they use to help surmount important or interesting problems. We welcome reader feedback on all of our columns and articles; please send your commentstoeic@clinorthop.org.

The author certifies that she, or any members of her immediate family, have no funding or commercial associations (eg, consultancies, stock ownership, equity interest, patent/ licensing arrangements, etc) that might pose a conflict of interest in connection with the submitted article.

All ICMJE Conflict of Interest Forms for authors and Clinical Orthopaedics and Related Research ${ }^{\mathbb{R}}$ editors and board members are on file with the publication and can be viewed on request.

The opinions expressed are those of the writers, and do not reflect the opinion or policy of Clinical Orthopaedics and Related Research $^{\mathbb{R}}$ or The Association of Bone and Joint Surgeons ${ }^{\circledR}$. dressing application represent the critical steps. Understanding the regional anatomy and knowing the pitfalls of the procedure are part of the entire intervention. Accordingly, I offer five quotes, primarily from mentors, which represent both the tenets and caprice of surgery.

1. “There's always a snake in the grass."

During residency, my chair Dr. Richard Burton frequently said this. No matter how confident you are, or how great the case is going, danger lurks. Although I generally favor a glass half-full approach, remembering this phrase keeps the mind alert to the anomalous reverse palmaris longus muscle in the carpal canal, the superior gluteal artery in a posterior iliac crest bone harvest, or tearing elderly skin while removing the plastic drape around a tourniquet at the end of the procedure.

2. On complications: "Ask me how I know."

Also in residency, Dr. John DeVanny often uttered this while

\section{A. L. Ladd MD ( $\square)$}

Chase Hand and Upper Limb Center, Stanford University, 770 Welch Rd. Suite 400, Palo Alto, CA 94304-1801, USA e-mail: alad@stanford.edu rolling his sage eyes. It typically followed a cautionary, "Watch your retraction on the piriformis," or, "Do you really want to hit that again?" Implied was, "I've seen-more than once-a sciatic nerve palsy/fractured femur from just that maneuver." Complications will happen. Be prepared enough to know and look out for the typical ones. As much as we would like to avoid them, complications remain opportunities for learning. With time and distance, like for Dr. DeVanny, they can be incorporated into humorous but important daily lessons.

3. "The purpose of an assistant is to make the surgeon look good." I first heard this quote from Dr. Norman Shumway, the great cardiac surgeon from Stanford University, who believed that the entire team was responsible for executed excellence in the operating theatre. All assistants-from the medical student, to the scrub tech, to the fellow eager to get the scalpel-are engaged. Knowing the steps of a procedure, understanding the critical and high-risk portions, and recognizing whether it is safe to change the position of a retractor on one's own or when 


\section{Pearls}

to ask for instruments ahead of time are all part of the culture of engagement. Complications have been avoided when a peripheral, but alert, resident grabbed the implant about to fall off the table, or the student asked why the tendon about to be harvested looked a lot like a nerve. Ask me how I know.

\section{4. "Be brief, be bright, be gone."}

I first heard this phrase while honing my presentation skills, but it equally applies to performing surgery. Thoughtful planning and mental rehearsal permits economy of motion. Automobile giant Walter Chrysler, in finding the simplest solutions for difficult tasks, is credited with saying, "Whenever there is a hard job to be done, I assign it to a lazy man; he is sure to find an easy way of doing it" [1]. Not that surgery should be a lazy task, but the most efficient way to do something saves time, money, potentially lives, and comes with practice and a conscious recognition of ways to economize. Placing a needlewhether a 10-0 suture in a tiny vessel, or a 2-0 in a large hip wound-without using the forceps to reload the needle driver is one example. Simply asking ahead for instruments is another. These measures, likely thought of as the simplest aspects of the procedure, begin to add up. If with scissor dissection you have made three spreads in the same region, you have duplicated the effort of two of those. Coordinated motion is swift, but not fast or sloppy.

\section{5. "Run through the tape."}

The high school coach reminds the sprinter to "run through the tape." Keep the heat on-especially when the end of the race is in sight. The final stage of surgery includes returning the patient's anatomy to its rightful owner, one that involves the entire anesthesia and surgical team. Placing a dressing that pads bony prominences and vulnerable peripheral nerves, and applying a compressive, but not restrictive cast or splint are as important as identifying these anatomical structures before an incision. Proper placement of drains and catheters, reestablishing limb and digit viability after a complex neurovascular repair or after the tourniquet is released, and providing a safe transition to the recovery room are all part of the final leg of our surgical performance.

No doubt you have heard the expression "better to be lucky than good," a tongue-in-cheek phrase we often apply to surgery. Perhaps the more accurate quote-at far as orthopaedic surgeons are concernedcomes from Louis Pasteur: "Chance favors the prepared mind."

\section{Reference}

1. Quote Investigator. Choose a lazy person to do a hard job because that person will find an easy way to do it. Available at: http://quoteinvestigator.com/2014/02/26/lazy-job/. Accessed August 11, 2016. 